\title{
Numerical Simulation of Porosity in Cements
}

\author{
Q. H. Do · S. Bishnoi · K. L. Scrivener
}

Received: 28 June 2012 / Accepted: 13 May 2013 / Published online: 24 May 2013

C Springer Science+Business Media Dordrecht 2013

\begin{abstract}
The pores in cementitious materials, their sizes and connectivity have an important influence on the durability of concrete. Several microstructural models have been developed to simulate the three-dimensional pore network in cement pastes. In this article, microstructures with the $\mu$ ic model are compared with experimental results. It is seen that despite having a resolution for the capillary pores very close to reality, the experimentally observed breakthrough diameter from mercury intrusion is much lower than the values obtained by applying an algorithm of mercury intrusion to the simulated microstructure. The effect of some of the most important input parameters on the pore sizes in the simulated microstructure explored. The phenomenon which seems best able to explain this discrepancy is that $\mathrm{C}-\mathrm{S}-\mathrm{H}$ is not in fact a phase with a smooth surface as represented in microstructural models, but a phase which grows as needles into the pore space, leading to very small water-filled capillary pores from quite young ages. The results demonstrate it will be extremely challenging to represent the porosity of real microstructures in microstructural models on the scale of hundreds of microns necessary to study macroscopic transport.
\end{abstract}

Keywords Modelling · Microstructure · Pore sizes · Mercury intrusion porosimetry · Cement hydration

\section{Introduction}

The transport of fluids through the capillary pore network is known to control the durability of concrete. This network is formed during the hydration of cement, when unhydrated phases react with water to form hydrates which increase the solid volume, filling the originally

Q. H. Do $(\bowtie) \cdot$ K. L. Scrivener

Laboratoire des Matériaux de Construction, Ecole Polytechnique Fédérale de Lausanne (EPFL),

Lausanne, Switzerland

e-mail: quanghuy.do@epfl.ch

S. Bishnoi

Department of Civil Engineering, Indian Institute of Technology Delhi, New Delhi, India 
water-filled space between the cement particles. This leads to a refinement in the capillary porosity and hence a reduction in the permeability of the material. Two main approaches have been developed to model the transport properties of hydrating cement pastes. In the first approach, transport is calculated by homogenising the microstructure using experimentally measured or calculated global values (e.g. Marchand et al. 2002). In the other approach, microstructural models are used to recreate the complex capillary pore network in cement pastes (Garboczi and Bentz 1991; Navi and Pignat 1996; Munch and Holzer 2008; Zhou et al. 2010). This article examines the ability of such microstructural models to accurately reproduce the capillary pore network of cementitious materials.

Microstructural models numerically simulate microstructural development and generate three-dimensional images of the microstructure at various stages of hydration. The images can then be used to predict macroscopically measurable properties such as elastic moduli (Haecker et al. 2005; Sanahuja et al. 2007; Pichler et al. 2009), rates of hydration (Bishnoi and Scrivener 2009b; Kumar et al. 2012), autogenous shrinkage (Koenders and van Breugel 1997) and permeability of cement pastes (Bentz et al. 1999). Microstructural models can themselves be classified into two main types according to the approach used to represent the microstructural information in the computer memory. In the discrete approach, the computational volume is divided into smaller finite-sized 'voxels' containing phase information that can evolve with hydration. The vector approach uses simple geometric shapes such as spheres and shells to represent the elements in the microstructure. The size of the smallest element in a discrete model is limited by the voxel size, but no such limitation exists in the vector approach. However, the characterisation of the pore space using the vector approach has been found to be computationally complex (Pignat et al. 2005; Bryant et al. 1993), so vector microstructures are typically converted to a discrete format to analyse their porosity.

To examine the accuracy of simulated microstructures they must be compared to experimental results. This is made difficult by the fact that all experimental techniques to determine the capillary pore structure of cementitious materials have limitations. Mercury intrusion porosimetry (MIP) continues to be the most widely used and has been shown to provide good comparisons among different systems. The main limitation of MIP is that it does not really measure pore 'size', but the volume of porosity which can be accessed through a given size of pore entry (Abell et al. 1999; Diamond 2010). In cementitious materials, the necks connecting pores are generally very small and a large volume (including much 'larger' pores) is accessed through small pore entries. (The term 'ink bottle effect', commonly used to describe this phenomenon, is misleading as it implies that it concerns only dead end pores.) For this reason, it is really not useful to consider the derivative MIP curve (a so-called pore size distribution). The cumulative curve does, however, provide useful information on the threshold pore entry size, below which the majority of the porosity becomes accessible, and the total intrudable porosity. Other criticisms of MIP are: that the technique requires prior removal of water, which may change the microstructure; that the high pressures used damage the microstructure (particularly at young ages) and that the mercury does not intrude all the porosity (particularly at late ages). Despite these drawbacks, we consider the technique as the best available to make quantitative comparisons with the output of microstructural models of capillary porosity, due the relative ease of measurement, its reproducibility and the fact that the physics behind the intrusion process are well understood.

The calculation of pore sizes from the numerical microstructures is also far from straightforward. Lin and Cohen (1982) first applied a method called morphological thinning, which is similar to skeletisation often used in image analysis, to quantify the geometry of microporous systems or models thereof. This technique is useful to describe the topology of digitised threedimensional images. Later, Baldwin et al. (1996) modified the method to characterise pore 
structures. Navi and Pignat (1999) applied this method to determine the pore-size distribution of cement pastes and to simulate MIP on such systems.

It has been reported that, due to the limited resolution in the discrete approach, simulations of mercury intrusion appear to show the microstructures to be much less connected than real measurements (Bentz and Martys 1994; Garboczi and Bentz 2001). On the other hand, previous work with the vector approach could not deal with the larger number of fine particles in real PSDs of anhydrous cements, which led to higher connectivity in the simulated microstructures than in reality (Pignat et al. 2005; Ye et al. 2003). With developments in computational capacities and methods both these limitations can now be reduced.

In this article, the $\mu$ ic microstructural modelling platform (Bishnoi and Scrivener 2009a), which uses the vector approach to generate three-dimensional microstructures, has been used to study the effect of various input parameters on the pore structures of simulated microstructures. Pore networks in the microstructures obtained from $\mu$ ic are discretised and analysed to calculate the total porosity and pore-size distributions. An algorithm is also applied to simulate the process of mercury intrusion. First, the total porosity in the simulations is compared with the porosity obtained from MIP measurements, then parameters such as the resolution of the microstructures, the roughness and shape of particles and the number of particles are then varied to study their effect on the breakthrough diameter.

We would like to stress that in this article, the simulations only represent explicitly, socalled, capillary pores, which are the spaces not occupied by hydration products. In the type of simulated microstructure considered here, with volumes of dimension around $100 \mu \mathrm{m}$, it is not possible to explicitly represent the, so-called, 'gel' pores with dimensions of a few nanometres, which are an intrinsic part of the $\mathrm{C}-\mathrm{S}-\mathrm{H}$. However, as discussed later, the amount of these gel pores will affect the 'bulk' density of the C-S-H. The gel pores are generally not considered to play a major role in transport.

\section{Numerical Modelling}

\subsection{Method to Model Pore Sizes}

In this article, the pore sizes are calculated using a slightly modified version of the method published earlier by Bishnoi and Scrivener (2009a). This method uses the technique of threedimensional erosion of pore space to locate pore centres and then the extent of each pore is calculated by walking back to the pore surfaces from each of the pore centres. Computationally, the speed of this method was increased by the introduction of lists that help in the erosion process. A parallel version of the programme was also developed, in which the volume is divided into smaller sub-volumes, each of which can be separately analysed on different processors and averaged to calculate the overall pore-size distribution. The accuracy of the subdivision technique is presented later in this article. This technique allowed calculation of pore sizes down to $10 \mathrm{~nm}$ in microstructures with $100 \mu \mathrm{m}$ dimension, using a total of one trillion $\left(10^{12}\right)$ voxels within a few days of computational time.

Figure 1 shows performance of the three versions of the algorithm for pore simulations of a Portland cement microstructure volume of size $100 \mu \mathrm{m}$ at $20 \%$ total porosity. In the figure, $O\left(n^{x}\right)$ means that computational time is proportional to the $x$ th power of the size of the problem $(n)$, i.e. the number of voxels in the computational volume. It can be seen in the figure that an improvement in the order of the problem was achieved through the singleprocessor optimisation described above, while a linear reduction in the computational time was achieved by parallelising over ten nodes. 
Fig. 1 Improvement in computational times using the improved approach. The order of the original model was $O\left(n^{2.33}\right)$ and for the other two versions is $O\left(n^{1.67}\right)$

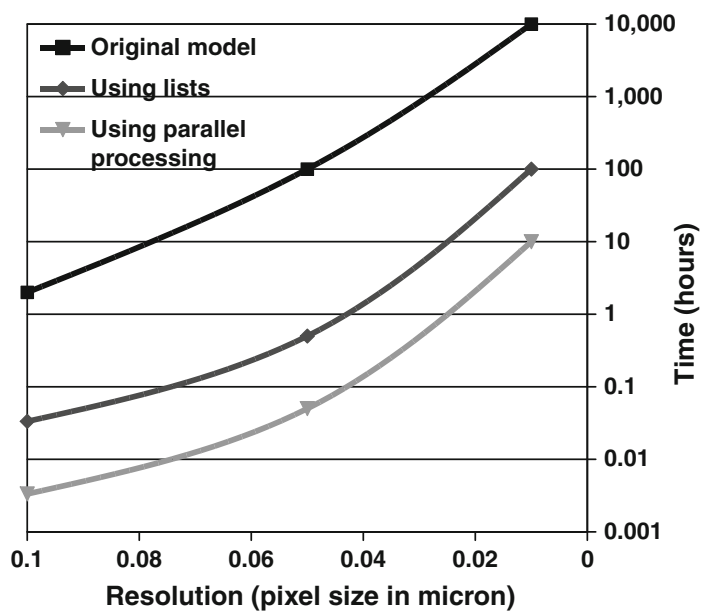

\subsection{Method to Model MIP}

The MIP curves of the simulated computational volume were calculated by mimicking the flow of mercury through the volume under pressure, automatically accounting for the pore connectivity and the 'pore entry effect'. Using the voxel-erosion method (Bishnoi and Scrivener 2009a), all pore voxels are first marked with their distance from the closest solid boundary (Fig. 2). In order to simulate the dependence of the intrusion diameter on the applied pressure, the process is simulated in several steps. In the first step, it is assumed that mercury flows only into the pores that are at least as large as the largest pore on the boundary of the computational volume. The pore sizes are identified using the distances obtained by erosion described in Sect. 2.1. Following the walk-back technique discussed above, the extent of the intruded pores is then identified. Flow into a voxel of a given pore size is allowed only if the voxel is either located on the boundary of the computational volume or if it shares a face with a voxel which has already been intruded. The iterative process of intrusion continues until all connected pores of at least the size being intruded have been filled. In each subsequent step, flow is allowed into pores that are one size smaller than those intruded in the previous step. The number of voxels intruded in any step is measured as the apparent volume for the respective pore size as obtained experimentally from MIP. The above process is also illustrated in Fig. 3 and the pore-entry size measured using this technique is shown in Fig. 4.

\section{Simulations}

The composition of the Portland cement simulated in this article is listed in Table 1 and its particle size distribution is shown in Fig. 5. A water-cement ratio of 0.35 was used for all simulations. The hydration kinetics of the cement were measured using isothermal calorimetry at $20^{\circ} \mathrm{C}$. MIP measurements were made on pastes hydrated for $6 \mathrm{~h}, 12 \mathrm{~h}$ and 3 days, corresponding to 8,23 and $60 \%$ hydration, respectively, based on the calorimetry measurements.

Hydration was simulated on a cubic computational volume of $100 \mu \mathrm{m}$ on each side. The size of the computational volume was chosen to be about 2.5 times larger than the largest cement grain. A total of 1,193,100 cement particles were generated using the known PSD of 

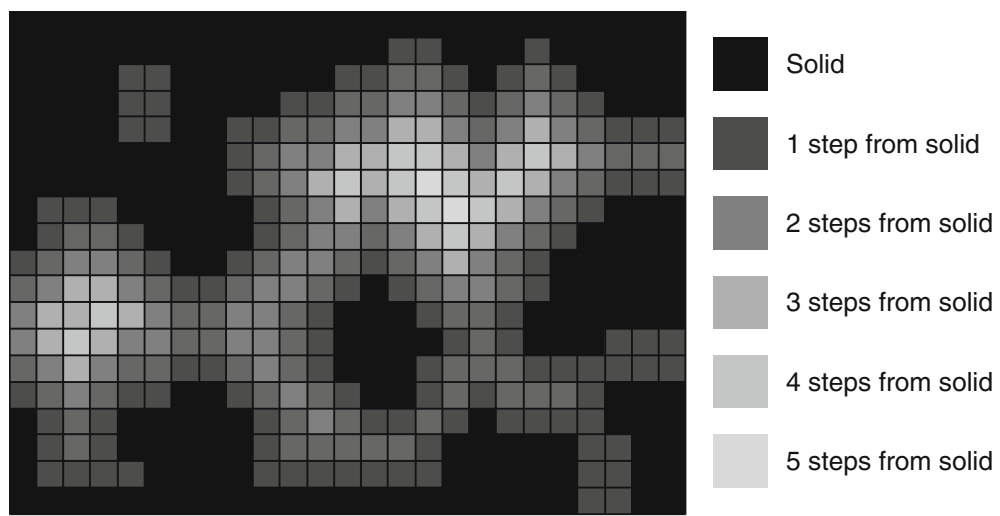

Fig. 2 Voxel erosion from the solid wall to find the pore centres. Grey region the solids, white regions the pores. Numbers the number of steps required to reach a cell from the solid boundary

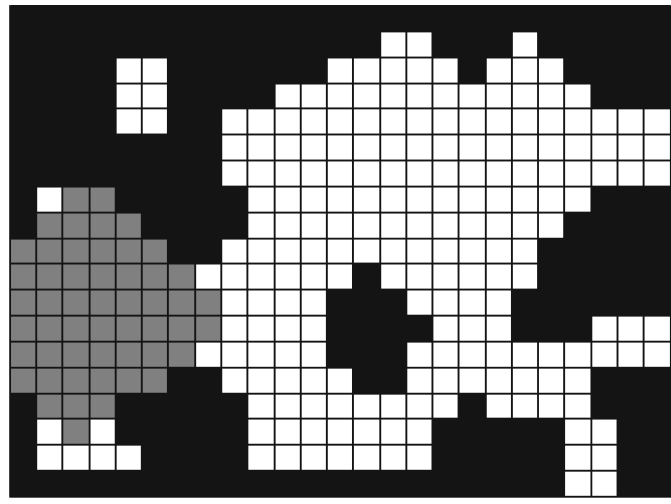

Solid

Intrusion for size 3

Fig. 3 Mercury fills the porosity after the first step of the liquid intrusion process. Grey cells the region that has been intruded by mercury in the first step of the MIP simulation
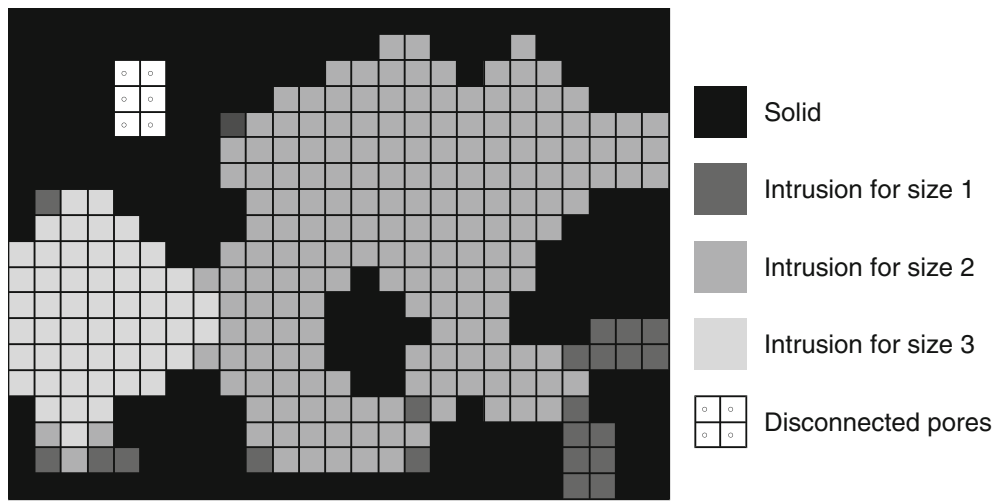

Fig. 4 The result from MIP simulation. All connected pores appear to be of sizes 1, 2 or 3 despite larger pores being present 
Table 1 Main compositions of the Portland cement

\begin{tabular}{llllll}
\hline & Alite & Belite & Aluminate & Ferrite & Gypsum \\
\hline Composition (\%) & 73.25 & 8.21 & 4.75 & 9.81 & 3.98 \\
\hline
\end{tabular}

Fig. 5 Particle size distribution used in the hydration model

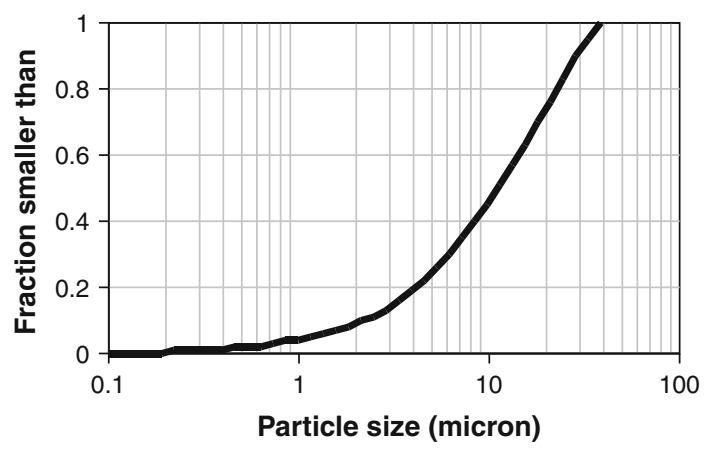

the cement. A description of the methodology used to generate and pack the particles and the implementation of the microstructural development model is discussed elsewhere (Bishnoi and Scrivener 2009a). Some of the hydration products, e.g. calcium silicate hydrate $(\mathrm{C}-\mathrm{S}-\mathrm{H})$ and ettringite are deposited around the hydrating cement particles and some others such as calcium hydroxide, also known as portlandite or $\mathrm{CH}$, nucleates in the pores. Microstructures were simulated corresponding to the measured degrees of hydration identified above. The hydration was carried out in 30 steps and the calculation took under $2 \mathrm{~h}$ on a desktop computer. The microstructures generated from the model were used to calculate pore sizes using the methods discussed earlier.

\subsection{Matching Total Porosity with Simulation Results}

Initial results from $\mu$ ic showed that if the bulk density of $\mathrm{C}-\mathrm{S}-\mathrm{H}$ is assumed to be a constant value of $2.0 \mathrm{~g} / \mathrm{cm}^{3}$, which is the typical value reported in literature (Jennings 2006), the total porosity calculated from the simulations was significantly higher than the MIP measurements at early ages. At early ages, when the pore structure is still well connected, it is reasonable to assume that all capillary pores are intruded in the MIP measurements and therefore the total volume of mercury intruded at the maximum pressure gives a good measure of the total volume of capillary pores in the system. In fact, at young ages it could be possible that the pressure of mercury damages the solid phases so, if anything, might over estimate the total porosity. It was found that in order to obtain the same porosities as the experiments, the bulk density of C-S-H had to be adjusted to $0.97,1.38$ and $1.97 \mathrm{~g} / \mathrm{cm}^{3}$, respectively, for degrees of hydration of 8, 23 and $60 \%$ (Fig. 6). The possibility of a variable density of C-S-H was conjectured earlier (Jennings 2004). Bishnoi and Scrivener (2009b) also postulated the idea of $\mathrm{C}-\mathrm{S}-\mathrm{H}$ densification during the course of hydration to explain the observed hydration kinetics. Recently, direct measurements of undried samples by proton NMR have confirmed that the 'bulk' density of C-S-H (including gel porosity) does increase during the course of hydration and the values obtained agree well with the values found here.

A comparison between the pore-size distribution and the mercury intrusion curves for the three microstructures (Fig. 7) shows that the entire pore network is connected at least 


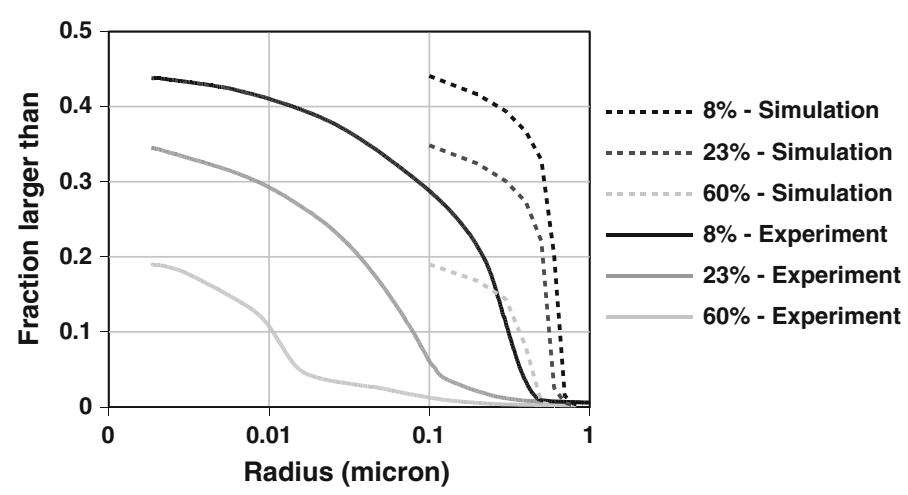

Fig. 6 Comparison of MIP simulations with experimental simulations at $6 \mathrm{~h}$ ( $8 \%$ hydration), $12 \mathrm{~h}(23 \%$ hydration) and 3 days (60\% hydration). The C-S-H density was set to $0.97 \mathrm{~g} / \mathrm{cm}^{3}$ at $6 \mathrm{~h}, 1.38 \mathrm{~g} / \mathrm{cm}^{3}$ at $12 \mathrm{~h}$ and $1.97 \mathrm{~g} / \mathrm{cm}^{3}$ at 3 days

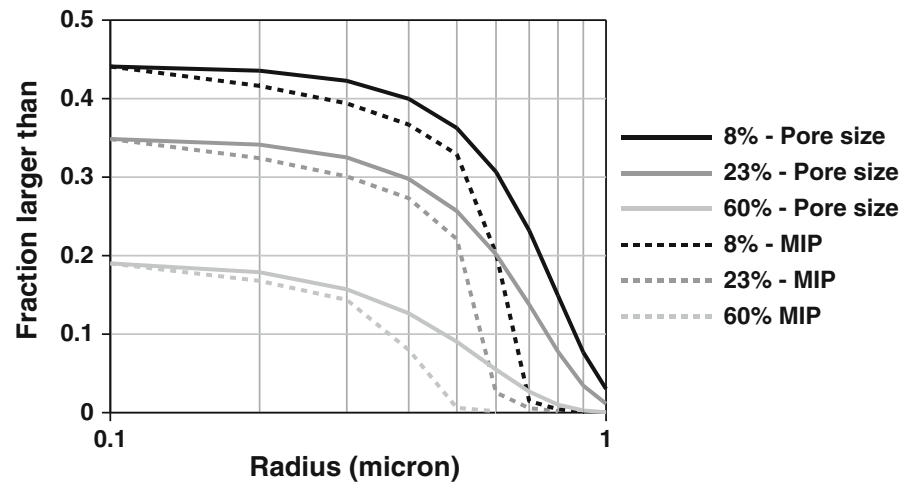

Fig. 7 Comparison of simulated pore-size distributions and MIPs for 8, 23 and $60 \%$ hydration shows that the entire pore network is percolated until $60 \%$ hydration

until $60 \%$ hydration. Even so the distribution of pore entry sizes in the simulated mercury intrusion curves shift to smaller sizes compared to the pore sizes. However, the breakthrough diameter in the mercury intrusion simulations was significantly higher than the experimental results. The reduction in the density of $\mathrm{C}-\mathrm{S}-\mathrm{H}$ did not significantly reduce the breakthrough diameter. The discrepancy between measured and simulated breakthrough diameters was found to increase as hydration progressed, with the experimental values decreasing by nearly one and a half orders of magnitude, while the simulated values were only halved.

The results above can interpreted to mean that either experimental MIP shows too low values of porosity and the breakthrough diameter or that the simulations give too high values compared to real microstructures. However, most artefacts which can be imagined for mercury intrusion experiments - for example the high pressures applied to the specimen leading to a collapse of the fine pores in $\mathrm{C}-\mathrm{S}-\mathrm{H}$-would be expected to increase the experimental determination of breakthrough diameter and total porosity rather than to decrease them. Only incomplete penetration of mercury into all the porosity could lead to the experimental volumes being too low, but incomplete penetration would be expected to be greater at high degrees of hydration, when the pores are more disconnected-which is not compatible with the observations. Therefore, we are led to the conclusion that the mercury intrusion results, 
do in fact, give a reasonable picture of the true pore structure (which is supported by the results of proton NMR published elsewhere []) and so that the simulated microstructures do not well represent the pore structure of real materials.

In order to understand the aspects of the microstructure which may be responsible for this discrepancy between the simulation and the reality, we investigated various parameters in the simulations, which might be expected to have a significant effect on the breakthrough diameter and therefore should potentially be changed to obtain a more realistic microstructure. Since the effect of density of $\mathrm{C}-\mathrm{S}-\mathrm{H}$ and generally the hydration products has already been seen, the value of the density of $\mathrm{C}-\mathrm{S}-\mathrm{H}$ was subsequently set to its expected long-term value of $2.0 \mathrm{~g} / \mathrm{cm}^{3}$. The results from these simulations are presented in the following sections.

\subsection{Impact of Simulation Parameters}

\subsubsection{Effect of Resolution}

As discussed above, the method used to characterise the pore sizes relies on a mesh of voxels superimposed on the vector microstructure. As it is possible to include smaller and a larger number of features in them, finer meshes tend to give better representations of the reality. However, this comes at the cost of higher computational memory and time requirements and practical limitations generally limit the maximum possible resolution in simulations. First, simulations were carried out on the same microstructure using different resolutions in order to study the effect of resolution on the threshold diameter and the pore-size distribution.

The resolution does not affect the total porosity due to the method of converting the vectorial simulations into digitised microstructures for analysis of the pore structure. The hydration and the resulting solid phases are generated by the vector approach which has no lower resolution. This vectorial representation is converted to a voxel representation, by sampling the phase at the centroid of that voxel. This sampling method is analogous to point counting, which gives a rigorously unbiased estimate of the volume fractions of different phase in a microstructure.

The higher resolutions ( 5 and $10 \mathrm{~nm}$ ) require calculations using the parallelisation. Therefore, the accuracy of this method was first tested on simulations using a relatively low resolution of $0.1 \mu \mathrm{m}$ by the single-processor and by the parallel versions of the programme. The calculations were carried out on microstructures having approximately $74 \%$ degree of hydration. The average of results from 1,000 parallel computations is compared with those obtained without sub-dividing the volume in Fig. 8. The results show the excellent accuracy of the method and confirm that it can be used to obtain pore-size calculations at higher resolutions.

Fig. 8 Accuracy test for the subdivision technique

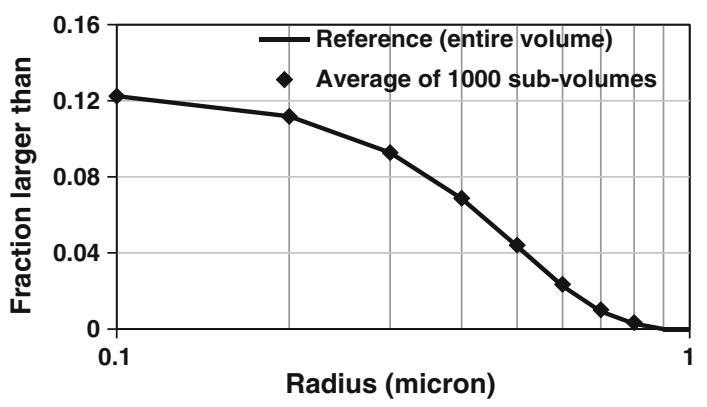

Radius (micron) 


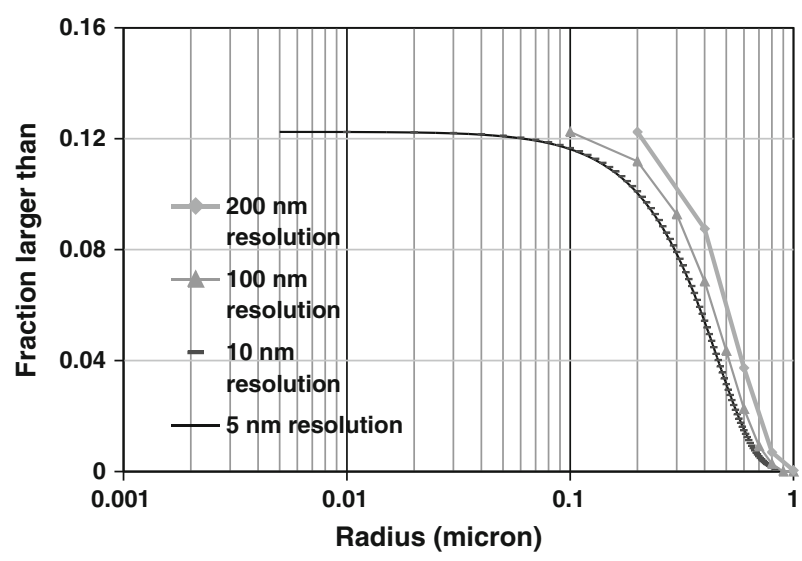

Fig. 9 Effect of resolution on pores size distribution

Figure 9 shows the pore-size distributions calculated from the microstructure using voxel sizes of 200, 100, 10 and $5 \mathrm{~nm}$. The simulations for the first two resolutions used the singleprocessor version of the code and the parallel version was used for the other two resolutions. The results, shown in Fig. 9, confirm that the resolution does not affect the total calculated porosity. The pore-size distributions become finer when finer resolutions are used, but converge at a voxel size of around $10 \mathrm{~nm}$. Although MIP simulations could not be carried out on the higher resolution microstructures due to computational limitations, the reduction in the pore sizes does not appear to be high enough to lead to an appreciable change in the MIP simulation results.

These results indicate that the observed increase in the threshold diameter is not due to the limited resolution of the microstructures.

\subsubsection{Effect of Size of Computational Volume on MIP Simulations}

In the MIP simulations, the intrusion is simulated from the boundary of the computational volume. However, as the computational volume represents only a small portion of a larger material, it is expected that the connectivity of pores will be reduced when larger computational volumes are used (Pignat 2003). This is because a larger number of the bigger pores are expected to be farther from the boundary, and so only accessible through finer pores, in the case of larger computational volumes. To study the effect of the size of the computational volume on the mercury intrusion curves, the computational volumes were placed next to each other to create larger cubes two and three times the size of the original volume. Due to the periodic boundary conditions used in $\mu$ ic, this yields a seamless and continuous microstructure.

A relatively higher voxel size of $0.2 \mu \mathrm{m}$ had to be used in these simulations as the number of voxels was too large when smaller voxel sizes were used with the computational volume that was 3 times the original volume. This increase in the voxel size is expected to reduce the degree of connectivity of pores and to further increase the pore-entry effect. A degree of hydration of $74 \%$ was chosen for the simulations as lower degrees of hydration would lead to microstructures with little pore entry effect and higher degrees of hydration would lead to pore structures that are too disconnected at the resolution used. 
Fig. 10 Effect of computational volume size on the MIP simulation

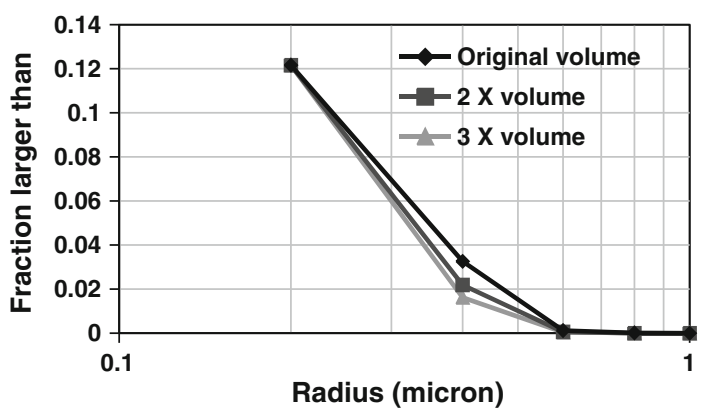

Results from MIP simulations on these 'multiplied' microstructures are shown in Fig. 10. The results show that the pores appear to be finer when larger microstructures are simulated even though the entire pore network is still connects at this above this resolution of $0.2 \mu \mathrm{m}$. However, no effect was observed on the breakthrough diameter, indicating that the deviation from the experimental results is not a result of the finite size of the computational volume being used.

\subsubsection{Effect of Flocculation}

It is generally considered that the flocculation of cement particles has a considerable effect on the properties of cement paste (Scherer et al. 2012a). As the default simulations in $\mu$ ic utilise a random parking algorithm for packing the initial particles in the computational volume, the effect of rearrangement of particles into chains on the pore-size distribution and MIP simulations was studied. Flocculated microstructures were obtained by following a two-step procedure where all particles in the system were first moved closer to the particle closest to them by half the original distance between them and were then rotated around the other particle either for a fixed number of steps in random directions or until they collided with a third particle. Although this method does not attempt to replicate any physical laws of particulate suspensions, it is observed that chains of particles are formed if the above steps are repeated 3-5 times. This technique had been originally developed in $\mu$ ic to allow a high density packing of particles (Bishnoi 2008). While other techniques for flocculation could lead to different results, the order of the difference measured is expected to be similar.

The effect of flocculation on pore size and MIP simulations at approximately 25 and $74 \%$ hydration are shown in Fig. 11. The results show that flocculation leads to an apparent increase in the pore entry size. This is because the rearrangement of particles into chains leads to slightly larger spaces between the chains. The results also show that the magnitude of the effect of flocculation reduces with hydration, as the extra space created due to flocculation is filled by the hydration products.

\subsubsection{Effect of Number of Nucleating Clusters}

Here, the effect of the number of nuclei of hydration products that create new particles in the pore space was studied. In the simulation of hydration, while most of the hydration products are assumed to grow concentrically over the surface of the cement particles, some of the products, e.g. Portlandite $(\mathrm{CH})$ form new particles in the pores. As few studies have attempted to study the factors that control the precipitation and growth of these clusters 


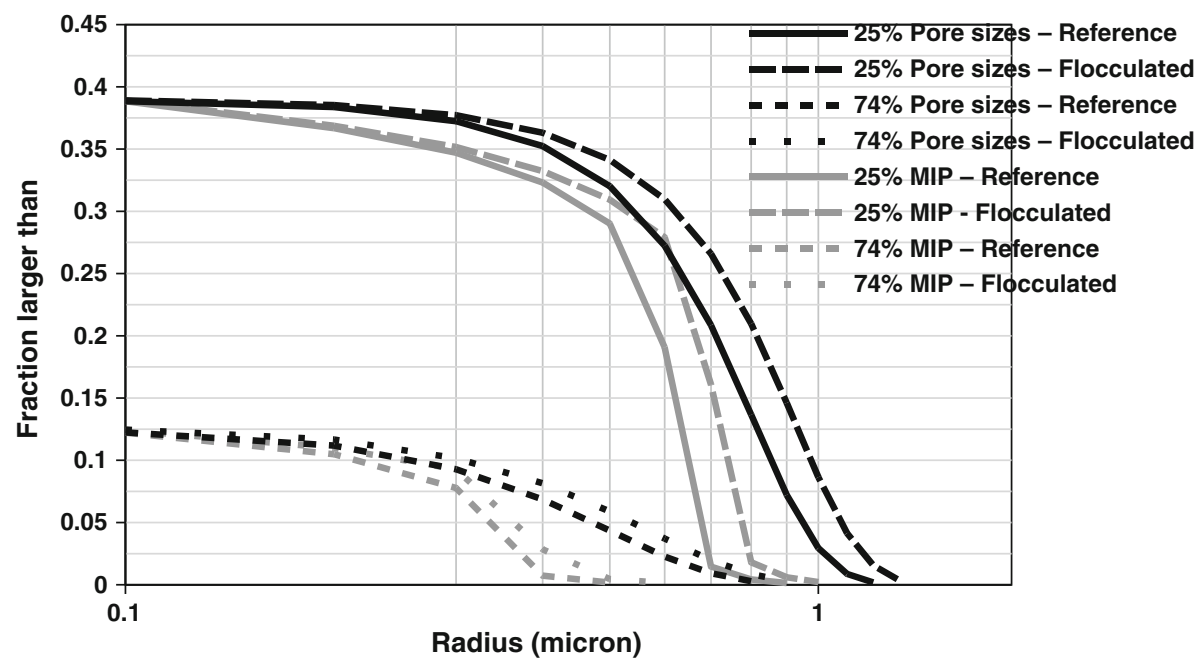

Fig. 11 Effect of flocculation on pore size and MIP simulations

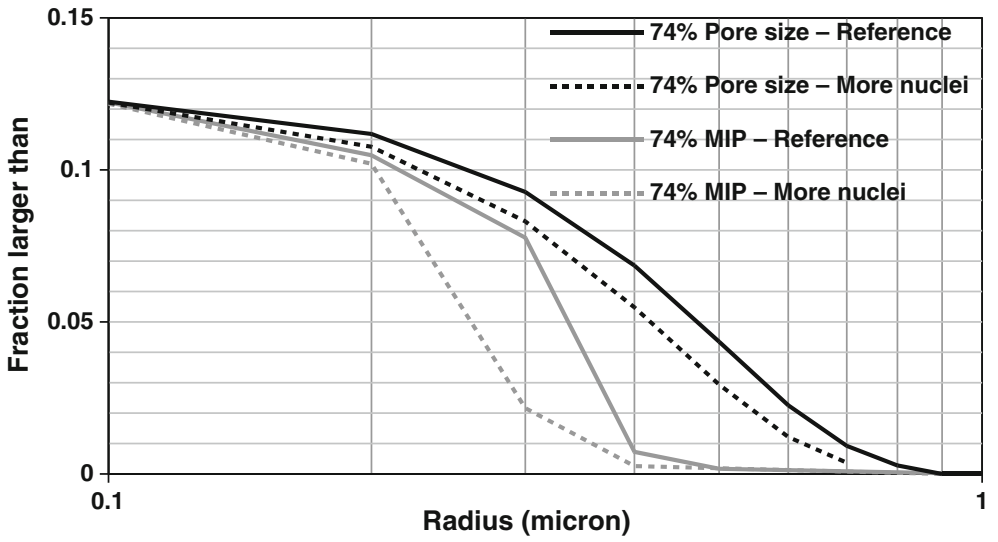

Fig. 12 Effect of number of new clusters nucleating on pore size and MIP simulations

(Gallucci and Scrivener 2007), the choice of the number of $\mathrm{CH}$ particles produced during hydration is often arbitrary (Navi and Pignat 1996) or based on empirical measurements with limited reliability (Jennings and Parrott 1986). Often, these particles are completely ignored and all hydration products are assumed to grow around the cement particles (van Breugel 1995). In order to test the sensitivity of the pore-size distribution and the threshold diameter to this parameter, the number of $\mathrm{CH}$ clusters in the simulation was increased from 5,000 to 400,000. The results in Fig. 12 show that there is a small reduction in the pore sizes and breakthrough diameter when a larger number of $\mathrm{CH}$ clusters are assumed. The number of clusters did not affect the total porosity in the microstructure as this is fixed by the relative volumes of reactants and products. The results demonstrate that the higher breakthrough diameter is not a result of the choice of the number of nucleating clusters. 


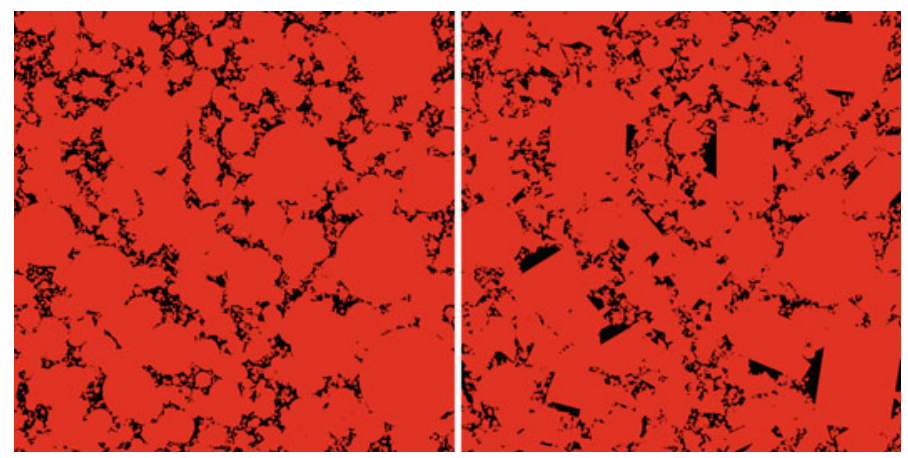

Fig. 13 Conversion of spherical particles to cylindrical particles (image at $75 \%$ hydration, dimension $100 \mu \mathrm{m})$. All solid phases are shown in red and the pores are shown in black

\subsubsection{Effect of Particle Shape}

One of the limitations of the vector approach is that due to computational restrictions it is difficult to represent the elements in the microstructure using shapes other than spheres. This is mainly due to the fact that it is easy to find the distance and detect overlaps between spheres. The accuracy of the vector microstructures has, therefore, been questioned and models that can consider more realistic shapes of particles have been developed (Bullard and Garboczi 2006; He et al. 2010). Simulations were carried out to study if the relatively higher breakthrough diameters simulated were a result of the spherical shape of the particles.

For these simulations, a microstructure generated from $\mu$ ic, having a degree of hydration of $74 \%$, was converted by replacing all spherical hydrated cement particles with cylinders. The cylindrical shape was chosen as its length to diameter ratio can be varied and it has sharp edges. We do not propose that cement grains are in fact spheres. No hydration was carried out on the cylindrical particles. The centres of the cylinders were made to coincide with the centre of the spheres in the original microstructure and the cylinders were randomly oriented. The outer radius of the cylinder was chosen to be the same as the outer radius of the original sphere. Although, theoretically, in order to have the same volume of the cylinder as the original sphere, the length of the cylinder should be 4/3rd its radius, it was found that as the pattern of overlap for cylinders is not the same as for spheres, the length of the cylinders had to be reduced by $7.5 \%$ in order to keep the total porosity the same as the original volume. During this process, the aspect ratio was kept constant for all cylinders. This modified microstructure was then meshed and pore-size and MIP simulations similar to those above were carried out. A comparison of the microstructures using spheres and cylinders is shown in Fig. 13. It can be seen in the figure that as the length of the cylinders is smaller than the diameter of the original sphere, larger pores may exist on the surface of some of the cylinders. This is an artefact of the conversion of the microstructure from spheres to cylinders and is not expected to occur if the growth of cylinders was simulated.

The comparison of results from cylindrical particles with those from spherical particles is shown in Fig. 14. The results show that a wider range of pore sizes are obtained when cylindrical particles are used and the breakthrough diameter appears to be higher. The results indicate that although the particle shape can have an effect on the pore sizes, the observed change is too small to account for the deviation from the experiments. 


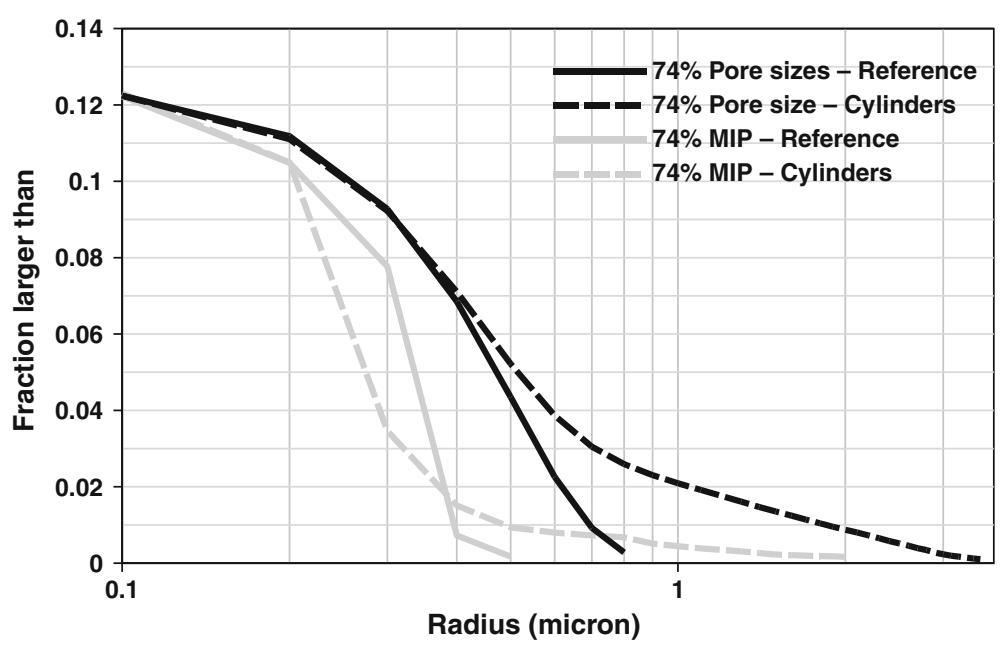

Fig. 14 Effect of particle shape on pore size and MIP simulations
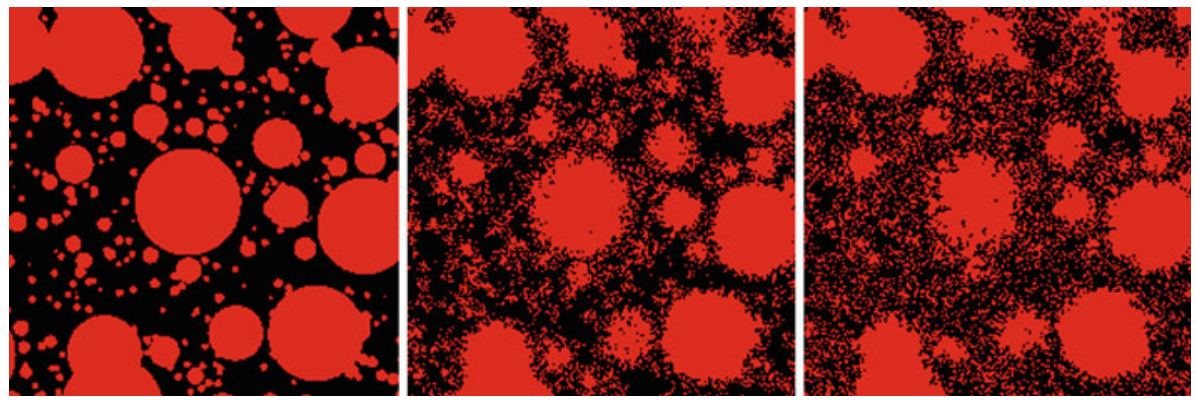

Fig. 15 The same region of the microstructure before and after 5 and 10 steps of roughening, respectively (from left to right, dimension $20 \mu \mathrm{m}$ ). All solid phases are shown in red and the pores are shown in black

\subsubsection{Effect of Surface Roughness}

Another limitation of the vector approach is that the surface of all pores is smooth. In real systems, it is expected that as the growth of hydration products will take place in a more random fashion, more rough surfaces will form and parts of the hydrates will extend into the pore space leading to a reduction in the effective pore size. In order to quantify this effect, a roughening algorithm was applied on the microstructures obtained from $\mu$ ic. This process was carried out in steps, where some of the pore voxels adjacent to solid voxels were converted to solid voxel in each step. The choice of the voxels to be converted is made by generating a pseudo-random number and comparing it with a pre-defined probability of conversion of $25 \%$ for each voxel. A similar process of randomly converting solid voxels to pore voxels was then carried out to obtain the same total porosity as the initial microstructure (Fig. 15). Again this 'roughening' process is rather arbitrary and just intended to get an idea of the effect it can have, rather than to be an accurate representation of a real microstrcuture.

A relatively lower degree of hydration of $35 \%$ was used in these simulations as the finer pore sizes at higher degrees of hydration did not allow sufficient number of steps of roughening to be carried out and the effect of roughening could not be seen clearly. The pore sizes and 


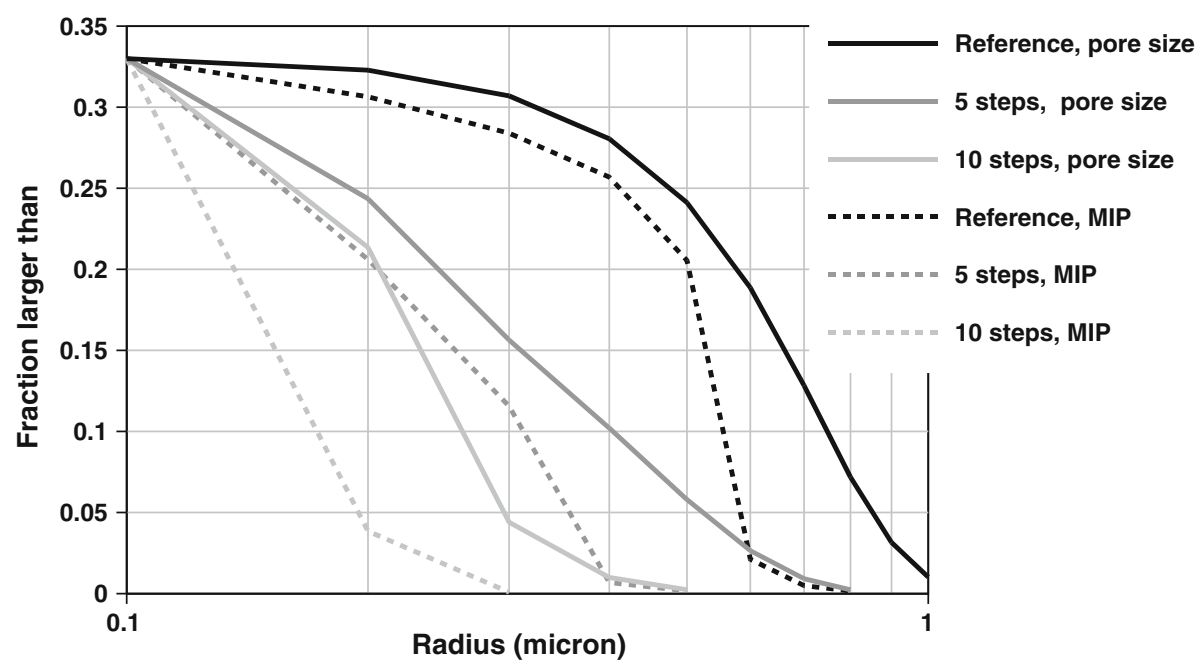

Fig. 16 Effect of surface roughness on pore size and MIP simulations

MIP simulations from the reference microstructure with no roughening are compared with microstructures with 5 and 10 steps of roughening in Fig. 16. It can be seen that the effect of roughness on pore sizes is the most significant of all the parameters considered and as expected rougher microstructures appear to have finer pore sizes.

It must be noted that in the case where 10 steps of roughening using voxels of $0.1 \mu \mathrm{m}$ were carried out, the maximum possible movement of the surface of the pore is $1 \mu \mathrm{m}$, which is close to the largest pore size. The average movement of the surface will be around $0.25 \mu \mathrm{m}$ in both directions due to a $25 \%$ possibility of conversion, making the pores smaller by approximately $0.5 \mu \mathrm{m}$. This change is reflected both in the largest pore size and the breakthrough diameter. This means that so as to reduce the breakthrough diameter by the order of magnitude needed to achieve agreement between experimental and simulation results, a roughness equivalent to over $90 \%$ the size of the largest pore would have to be introduced.

\section{Discussion of Results and the 'Nature of $\mathrm{C}-\mathrm{S}-\mathrm{H}$ '}

The results in this article demonstrate that while the pore-size distributions and MIP simulations on numerically generated microstructures are sensitive to parameters such as the density of the product, the number of particles and the roughness of the surface, the pore network in the microstructures simulated using numerical models is significantly different from the reality. The biggest difference between the two appears to be the much smaller breakthrough diameter in real systems.

Although most of the parameters considered in this study have some effect on the simulations, the only parameter that appeared to have an effect large enough on the breakthrough diameter to bring it close to the real values is the surface roughness. This indicates that the hydration product would have to extend into the pores by at least $90 \%$ of their size even at low degrees of hydrations. This result is in line with the observation, that in order to match the total porosities measured using MIP, the density of $\mathrm{C}-\mathrm{S}-\mathrm{H}$ during early ages has to be assumed to be less than half of its final value. Several studies have postulated a variable 
packing density of C-S-H (Jennings 2004; Masoero et al. 2012) and conclusions similar to those in this study were earlier drawn during a study of the hydration kinetics of alite (Bishnoi and Scrivener 2009b). These results were independently confirmed later in other studies (Thomas et al. 2009; Scherer et al. 2012a,b). Recent results measuring the pore sizes on hydrating cement pastes that have never been dried (Mueller et al. 2013) confirm the absence of pores larger than a few tens of nanometres and therefore spread of the hydration products throughout a large part of the microstructure after $24 \mathrm{~h}$ of hydration. Similar results, although without quantification, have been reported earlier (Fratini et al. 2003; McDonald et al. 2007). It must be noted that although this $\mathrm{C}-\mathrm{S}-\mathrm{H}$ is often referred to as a 'loosely packed product' at least some of this product has to be strong enough to withstand the pressures applied during MIP measurements in order to explain the results in this study. Furthermore, the apparent bulk density of $\mathrm{C}-\mathrm{S}-\mathrm{H}$ in this study may be higher than the real values due to a partial collapse of the loose product.

The results also demonstrate that in order to obtain more a realistic representation of the microstructural development in cement pastes, a proper treatment of this product is required in the microstructural models. It is expected that a better understanding of this 'diffuse' growth of $\mathrm{C}-\mathrm{S}-\mathrm{H}$ through experiments and simulations will significantly affect our current understanding of the way the properties of concrete develop.

\section{Conclusions}

A new simulation algorithms based on the voxel method was proposed to simulate mercury intrusion curves of numerical microstructures of cementitious systems at higher resolutions than previously possible. It was also shown that through parallelisation and the use of sufficiently small voxels, an accurate characterisation of numerical microstructures can be carried out.

The sensitivity of the calculated pore-size distributions to various parameters used in microstructural simulations was studied. Most importantly, it was seen that a more diffuse growth of $\mathrm{C}-\mathrm{S}-\mathrm{H}$ has to be considered in order to obtain better agreement between the experiments and the simulations. First, a lower bulk density of $\mathrm{C}-\mathrm{S}-\mathrm{H}$ has to be assumed at early age to obtain an agreement between the experimentally measured and simulated total porosity. Then, even at early ages, $\mathrm{C}-\mathrm{S}-\mathrm{H}$ must be distributed throughout a major portion of the capillary pores, with 'rough' edges-for example needles projecting into the empty pores - in order to obtain better estimates of the breakthrough diameter.

While it is admitted that the approach used to quantify pore sizes is approximate, the errors due to the simplified approach used are expected to be much smaller than the difference between computed and experimental curves. It can finally be concluded that the currently available microstructural simulations, in which $\mathrm{C}-\mathrm{S}-\mathrm{H}$ is assumed to have compact growth with a smooth interface with the porosity, give unrealistic representations of the pore structure of cement paste.

\section{References}

Abell, A.B., Willis, K.L., Lange, D.A.: Mercury intrusion porosimetry and image analysis of cement-based materials. J. Colloid Interface Sci. 211, 39-44 (1999)

Baldwin, C.A., Sederman, A.J., Mantle, M.D., Alexander, P., Gladden, L.F.: Determination and characterization of the structure of a pore space from 3D volume images. J. Colloid Interface Sci. 181, 79-92 (1996) 
Bentz, D.P., Martys, N.S.: Hydraulic radius and transport in reconstructed model three-dimensional porous media. Transp. Porous Media 17, 221-238 (1994)

Bentz, D.P., Garboczi, E.J., Haecker, C.J., Jensen, O.M.: Effects of cement particle size distribution on performance properties of Portland cement-based materials. Cem. Concr. Res. 29, 1663-1671 (1999)

Bishnoi, S.: Vector modelling of hydrating cement microstructure and kinetics. Doctoral Thesis, EPFL, Switzerland (2008)

Bishnoi, S., Scrivener, K.L.: A new platform for modelling the hydration of cements. Cem. Concr. Res. 39, 266-274 (2009)

Bishnoi, S., Scrivener, K.L.: Studying nucleation and growth kinetics of alite hydration using $\mu$ ic. Cem. Concr. Res. 39, 849-860 (2009)

Bryant, S.L., King, P.R., Mellor, D.W.: Network model evaluation of permeability and spatial correlation in a real random sphere packing. Transp. Porous Media 11, 53-70 (1993)

Bullard, J.W., Garboczi, E.J.: A model investigation of the influence of particle shape on Portland cement hydration. Cem. Concr. Res. 36, 1007-1015 (2006)

Diamond, S.: Mercury porosimetry: an inappropriate method for the measurement of pore-size distributions in cement-based materials. Cem. Concr. Res. 30, 1517-1525 (2010)

Fratini, E., Chen, S.-H., Baglioni, P.: Investigation of the temporal evolution of translational dynamics of water molecules in hydrated calcium aluminate pastes. J. Phys. Chem. B 107, 10057-10062 (2003)

Gallucci, E., Scrivener, K.: Crystallisation of calcium hydroxide in early age model and ordinary cementitious systems. Cem. Concr. Res. 37, 492-501 (2007)

Garboczi, E.J., Bentz, D.P.: Digitized simulation of mercury intrusion porosimetry. Adv. Cem. Mater. 16, 365-380 (1991)

Garboczi, E.J., Bentz, D.P.: The effect of statistical fluctuation, finite size error, and digital resolution on the phase percolation and transport properties of the NIST cement hydration model. Cem. Concr. Res. 31, 1501-1514 (2001)

Haecker, C.-J., Garboczi, E.J., Bullard, J.W., Bohn, R.B., Sun, Z., Shah, S.P., Voigt, T.: Modeling the linear elastic properties of Portland cement paste. Cem. Concr. Res. 35, 1948-1960 (2005)

He, H., Guo, Z., Stroeven, P., Stroeven, M., Sluys, L.J.: Strategy on simulation of arbitrary shaped cement grains in concrete. Image Anal. Stereol. 29, 79-84 (2010)

Jennings, H.M.: Colloid model of $\mathrm{C}-\mathrm{S}-\mathrm{H}$ and implications to the problem of creep and shrinkage. Mater. Struct. 37, 59-70 (2004)

Jennings, H.M., Parrott, L.J.: Microstructural analysis of hardened alite paste, part II: microscopy and reaction products. J. Mater. Sci. 21, 4053-4059 (1986)

Koenders, E.A.B., van Breugel, K.: Numerical modelling of autogenous shrinkage of hardening cement paste. Cem. Concr. Res. 27, 1489-1499 (1997)

Kumar, A., Bishnoi, S., Scrivener, K.L.: Modelling early age hydration kinetics of alite. Cem. Concr. Res. 42, 903-918 (2012)

Lin, C., Cohen, M.H.: Quantitative methods for microgeometric modelling. J. Appl. Phys. 53, 4152-4165 (1982)

Marchand, J., Samson, E., Maltais, Y., Lee, R.J., Sahu, S.: Predicting the performance of concrete structures exposed to chemically aggressive environment-field validation. Mater. Struct. 35, 623-631 (2002)

Masoero E., Del Gado E., Pellenq R. J.-M., Ulm F.-J., Yip S.:Nanostructure and nanomechanics of cement: polydisperse colloidal packing. Phys. Rev. Lett. 109, 155503 (2012)

McDonald, P.J., Mitchell, J., Mulheron, M., Monteilhet, L., Korb, J.-P.: Two-dimensional correlation relaxation studies of cement pastes. Magn. Reson. Imaging 25, 470-473 (2007)

Mueller, A.C.A., Scrivener, K.L., Gajewicz, A.M., McDonald, P.J.: Densification of C-S-H measured by ${ }^{1}$ H NMR Relaxometry. J. Phys. Chem. 117, 403-412 (2013)

Munch, B., Holzer, L.: Contradicting geometrical concepts in pore size analysis attained with electron microscopy and mercury intrusion. J. Am. Ceram. Soc. 91, 4059-4067 (2008)

Navi, P., Pignat, C.: Simulation of cement hydration and the connectivity of the capillary pore space. Adv. Cem. Based Mater. 4, 58-67 (1996)

Navi, P., Pignat, C.: Three-dimensional characterization of the pore structure of a simulated cement paste. Cem. Concr. Res. 29, 507-514 (1999)

Pichler, B., Hellmich, C., Eberhardsteiner, J.: Spherical and acicular representation of hydrates in a micromechanical model for cement paste: prediction of early-age elasticity and strength. Acta Mech. 203, 137-162 (2009)

Pignat, C.: Simulation numérique de l'hydration du silicate tricalcique, charactérisation de la structure poreuse et de la perméabilité. Doctoral Thesis, École Polytechnique Fédérale de Lausanne (2003)

Pignat, C., Navi, P., Scrivener, K.: Simulation of cement paste microstructure hydration, pore space characterization and permeability determination. Mater. Struct. 38, 459-466 (2005) 
Sanahuja, J., Dormieux, L., Chanvillard, G.: Modelling elasticity of a hydrating cement paste. Cem. Concr. Res. 37, 1427-1439 (2007)

Scherer, G.W., Zhang, J., Thomas, J.J.: Nucleation and growth models for hydration of cement. Cem. Concr. Res. 42, 982-993 (2012)

Scherer, G.W., Zhang, J., Quintanilla, J.A., Torquato, S.: Hydration and percolation at the setting point. Cem. Concr. Res. 42, 665-672 (2012)

Thomas, J.J., Allen, A.J., Jennings, H.M.: Hydration kinetics and microstructure development of normal and $\mathrm{CaCl}_{2}$-accelerated tricalcium silicate pastes. J. Phys. Chem. 113, 19836-19844 (2009)

van Breugel, K.: Numerical simulation of hydration and microstructural development in hardening cementbased materials (I): theory. Cem. Concr. Res. 25, 319-331 (1995)

Ye, G., van Breugel, K., Fraaij, A.L.A.: Three-dimensional microstructure analysis of numerically simulated cementitious materials. Cem. Concr. Res. 33, 215-222 (2003)

Zhou, J., Ye, G., van Breugel, K.: Characterization of pore structure in cement-based materials using pressurization-depressurization cycling mercury intrusion porosimetry (PDC-MIP). Cem. Concr. Res. 40, 1120-1128 (2010) 